\title{
ON COMPACT HYPERSURFACES WITH CONSTANT SCALAR CURVATURE IN THE EUCLIDEAN SPACE
}

\author{
TAKASHI OKAYASU
}

Dedicated to Professors H. Mori and Y. Watanabe for their 60th birthdays

\begin{abstract}
Let $M$ be a compact hypersurface in the Euclidean space with cohomogeneity 2. We show that if the scalar curvature is constant, then $M$ is a standard sphere. This gives a partial positive answer to a problem presented by Yau.
\end{abstract}

\section{Introduction}

In the famous problem section of the book [14], Yau posed the following problem:

YAU's PROBLEM. Classify compact hypersurfaces in $\mathbf{R}^{N+1}$ which have constant scalar curvature. Are they isometric to $S^{N}$ ? If they are convex, then the answer is yes and was proved by Cheng-Yau.

In 1988, Ros [10] solved this problem affirmatively when the hypersurfaces are embedded. So there remains the case when the hypersurfaces are immersed. This situation is very similar to the Hopf's conjecture about constant mean curvature hypersurfaces. Hopf's conjecture asks whether compact hypersurfaces $M$ in $\mathbf{R}^{N+1}$ which have constant mean curvature are only round spheres. When the hypersurfaces are embedded, Aleksandrov [1] proved $M$ is a sphere in 1958. 25 years later, Hsiang-Teng-Yu [5] constructed nonspherical compact immersed hypersurfaces with constant mean curvature of dimension $N \geq 3$ and solved the conjecture negatively (soon after this, Wente [13] constructed an immersed torus with constant mean curvature in $\mathbf{R}^{3}$ ). The technic they used in [5] is the method of equivariant differential geometry. They assume a large isometry group acts on $M$, and reduce the constant mean curvature equation to a system of ordinary differential equations.

2000 Mathematics Subject Classification. Primary 53C40; Secondary 53C42.

Key words and phrases. scalar curvature, equivariant differential geometry.

Partially supported by Grant-in-Aid for Scientific Research (No. 15540057), Japan Society for the Promotion of Science.

Received December 1, 2004; revised May 13, 2005. 
It is natural to try to construct immersed compact hypersurfaces with constant scalar curvature by using the same method. But unfortunately, we cannot expect such an example to exist because the following theorem holds which is the main result of this paper.

THEOREM 1.1. Let $M$ be a generalized rotational closed hypersurface in the Euclidean space with cohomogeneity 2. If the scalar curvature is constant, then $M$ is a standard sphere.

To end this introduction we cite a recent paper of Cheng [2] which proved that Yau's conjecture is true in the class of compact oriented locally conformally flat hypersurfaces in $\mathbf{R}^{n+1}$.

The author would like to express his hearty thanks to the referee for indicating several mistakes.

\section{Preliminaries}

Let $\left(G, \Phi, \mathbf{R}^{n+1}\right)$ be an orthogonal transformation group with codimension two principal orbit type. They are classified completely by Hsiang-Lawson [4]. They are exactly those isotropy representations of symmetric spaces of rank 2 . Following [3] we give some basic facts.

(i) There exists a 2-dimensional linear subspace, $\mathbf{R}^{2}$, which is the fixed point of a chosen principal isotropy subgroup $H$ of $\left(G, \mathbf{R}^{n+1}\right)$ and intersects every $G$-orbit perpendicularly.

(ii) The Weyl group, $W=N(H, G) / H$, acts on $\mathbf{R}^{2}$ as a group generated by reflections and $\mathbf{R}^{n+1} / G \simeq \mathbf{R}^{2} / W$. Therefore, the orbit space $\mathbf{R}^{n+1} / G$ can be identified with the Weyl chamber of $\left(W, \mathbf{R}^{2}\right)$ and the orbital metric is flat, namely, a linear cone of angle $\pi / d, d=1,2,3,4$ or 6 .

Let $\gamma=\gamma(s)=(x(s), y(s))$ be a curve in $\mathbf{R}^{2} / W(s:$ arc-length $)$. Let $M_{\gamma}$ be a $G$-invariant hypersurface generated by $\gamma$. We call this hypersurface a generalized rotational hypersurface with cohomogeneity 2. We have the following lemma [5] which reduce the computation of the mean curvature of $M_{\gamma}$ to that of $\gamma$ in $\mathbf{R}^{2} / W$.

LEMMA 2.1.

$$
n H=x^{\prime} y^{\prime \prime}-y^{\prime} x^{\prime \prime}-\frac{\partial}{\partial v_{\xi}} \log \varphi,
$$

where $\varphi(x, y)$ is the volume of the principal orbit through $(x, y), \xi$ is the unit normal vector of $\gamma$ in $\mathbf{R}^{2} / W$ such that $\left\{\gamma^{\prime}, \xi\right\}$ has a positive orientaition, and $\frac{\partial}{\partial v_{\xi}}$ is the directional derivative in the direction $\xi$, that is, $\frac{\partial}{\partial v_{\xi}}=-y^{\prime} \frac{\partial}{\partial x}+x^{\prime} \frac{\partial}{\partial y}$. H is the mean curvature of $M_{\gamma}$ in the direction $v$ which projects to $\xi$.

To compute the scalar curvature, we use the following variational formula of Reilly [9] (see also [11]). 
Lemma 2.2. Let $M$ be an oriented hypersurface in $\mathbf{R}^{n+1}$. Let $v$ be $a$ unit normal vector field normal to $M$ and let $V=f v$ be a vector field along $M$ for some smooth function $f$ on $M$. Let $\Psi_{t}: M \rightarrow \mathbf{R}^{n+1}$ be a variation of $M$ with $\Psi_{0}(x)=x$ for $x \in M$ and $d \Psi_{t}(x) /\left.d t\right|_{t=0}=V(x)$, for all $x \in M$. Then

$$
\left.\frac{d}{d t}\right|_{t=0} \int_{M} n H d v=-\int_{M} f S d v
$$

where $H$ and $S$ are the mean curvature and the scalar curvature of $M$ respectively.

Now we have the following useful lemma.

Lemma 2.3. The scalar curvature $S$ of $M_{\gamma}$ is given by

$$
S=-2\left(x^{\prime} y^{\prime \prime}-y^{\prime} x^{\prime \prime}\right) \frac{\partial}{\partial v_{\xi}} \log \varphi+\left(\frac{\partial}{\partial v_{\xi}} \log \varphi\right)^{2}+\frac{\partial^{2}}{\partial v_{\xi}^{2}} \log \varphi .
$$

Proof. Let $f(s)$ be a function on $\gamma$ with compact support. We can naturally consider $f$ as a function on $M_{\gamma}$. Set $\gamma_{t}(s) \equiv(\bar{x}(s, t), \bar{y}(s, t)) \equiv(x(s), y(s))+$ tf $(s)\left(-y^{\prime}(s), x^{\prime}(s)\right)$ and consider a $G$-invariant hypersurface $M_{\gamma_{t}}$ generated by $\gamma_{t}$. Thus we have a variation $\Psi_{t}: M \rightarrow \mathbf{R}^{n+1}$ satisfying $\Psi_{0}(M)=M_{\gamma}$ and $d \Psi_{t} /\left.d t\right|_{t=0}=f v$. Note that $s$ is not an arclength parameter except for $t=0$. From Lemma 2.1 (or from the first variational formula of area) we obtain

$$
n H(t)=\frac{\bar{x}^{\prime} \bar{y}^{\prime \prime}-\bar{y}^{\prime} \bar{x}^{\prime \prime}}{\left\{\bar{x}^{\prime 2}+\bar{y}^{\prime 2}\right\}^{3 / 2}}-\frac{1}{\sqrt{\bar{x}^{\prime 2}+\bar{y}^{\prime 2}}}\left(-\bar{y}^{\prime} \frac{\partial}{\partial x}+\bar{x}^{\prime} \frac{\partial}{\partial y}\right) \log \varphi,
$$

where $H(t)$ is the mean curvature of $M_{\gamma_{t}}$ and we used "'" for differentiation with respect to $s$. Therefore

$$
\begin{aligned}
\left.\frac{d}{d t}\right|_{t=0} \int_{M} n H(t) d v= & \left.\int_{\gamma} \frac{d}{d t}\right|_{t=0}\left\{n H(t) \varphi\left(\gamma_{t}\right)\right\} d s \\
= & \int_{\gamma}\left[\left.\frac{d}{d t}\right|_{t=0}\{n H(t)\} \varphi(\gamma)+\left.n H \frac{d}{d t}\right|_{t=0}\left\{\varphi\left(\gamma_{t}\right)\right\}\right] d s \\
= & \int_{\gamma}\left[\left\{\left.\frac{d}{d t}\right|_{t=0} \frac{\bar{x}^{\prime} \bar{y}^{\prime \prime}-\bar{y}^{\prime} \bar{x}^{\prime \prime}}{\left\{\bar{x}^{\prime 2}+\bar{y}^{\prime 2}\right\}^{3 / 2}}\right\} \varphi\right. \\
& -\left\{\left.\frac{d}{d t}\right|_{t=0} \frac{1}{\sqrt{\bar{x}^{\prime 2}+\bar{y}^{\prime 2}}}\right\}\left(-\bar{y}^{\prime} \frac{\partial}{\partial x}+\bar{x}^{\prime} \frac{\partial}{\partial y}\right) \log \varphi \cdot \varphi \\
& -\left(-\left.\frac{d}{d t}\right|_{t=0} \bar{y}^{\prime} \frac{\partial}{\partial x}+\left.\frac{d}{d t}\right|_{t=0} \bar{x}^{\prime} \frac{\partial}{\partial y}\right) \log \varphi \cdot \varphi \\
& \left.-\left.\left(-y^{\prime} \frac{\partial}{\partial x}+x^{\prime} \frac{\partial}{\partial y}\right) \frac{d}{d t}\right|_{t=0} \log \varphi \cdot \varphi-f \cdot(n H)^{2} \cdot \varphi\right] d s
\end{aligned}
$$


because $s$ is the arclength of $\gamma$. Now we compute each terms of the right hand side of (2.5) separately. By using $\left(x^{\prime}\right)^{2}+\left(y^{\prime}\right)^{2}=1, x^{\prime} x^{\prime \prime}+y^{\prime} y^{\prime \prime}=0$ we obtain

$$
\begin{gathered}
\left.\frac{d}{d t}\right|_{t=0}\left\{\bar{x}^{\prime} \bar{y}^{\prime \prime}-\bar{y}^{\prime} \bar{x}^{\prime \prime}\right\} \\
=\left(\left.\frac{d}{d t}\right|_{t=0} \bar{x}^{\prime}\right) \bar{y}^{\prime \prime}+\overline{x^{\prime}}\left(\left.\frac{d}{d t}\right|_{t=0} \bar{y}^{\prime \prime}\right)-\left(\left.\frac{d}{d t}\right|_{t=0} \bar{y}^{\prime}\right) \bar{x}^{\prime \prime}-\bar{y}^{\prime}\left(\left.\frac{d}{d t}\right|_{t=0} \bar{x}^{\prime \prime}\right) \\
=-\left(f y^{\prime}\right)^{\prime} y^{\prime \prime}+x^{\prime}\left(f x^{\prime}\right)^{\prime \prime}-\left(f x^{\prime}\right)^{\prime} x^{\prime \prime}+y^{\prime}\left(f y^{\prime}\right)^{\prime \prime}, \\
\left.\frac{d}{d t}\right|_{t=0}\left\{\bar{x}^{\prime 2}+\bar{y}^{\prime 2}\right\}=-2 f\left(x^{\prime} y^{\prime \prime}-y^{\prime} x^{\prime \prime}\right) .
\end{gathered}
$$

Therefore by using integration by parts we get

(2.8) The first term of R.H.S. of (2.5)

$$
\begin{aligned}
=\int_{\gamma}\{ & -f\left\{\left(x^{\prime \prime}\right)^{2}+\left(y^{\prime \prime}\right)^{2}\right\}+x^{\prime}\left(f x^{\prime}\right)^{\prime \prime}+y^{\prime}\left(f y^{\prime}\right)^{\prime \prime} \\
& \left.+3 f\left(x^{\prime} y^{\prime \prime}-y^{\prime} x^{\prime \prime}\right)^{2}\right\} \varphi d s \\
=\int_{\gamma}[ & -2 f\left\{\left(x^{\prime \prime}\right)^{2}+\left(y^{\prime \prime}\right)^{2}\right\} \varphi-f^{\prime}\left(\varphi_{x} x^{\prime}+\varphi_{y} y^{\prime}\right) \\
& \left.+3 f\left(x^{\prime} y^{\prime \prime}-y^{\prime} x^{\prime \prime}\right)^{2} \varphi\right] d s .
\end{aligned}
$$

(2.9) The 2 nd term of R.H.S. of (2.5)

$$
\begin{aligned}
& =\int_{\gamma}-f\left(x^{\prime} y^{\prime \prime}-y^{\prime} x^{\prime \prime}\right) \frac{\partial}{\partial v_{\xi}} \log \varphi \cdot \varphi d s \\
& =\int_{\gamma}-f\left(x^{\prime} y^{\prime \prime}-y^{\prime} x^{\prime \prime}\right)\left(-y^{\prime} \varphi_{x}+x^{\prime} \varphi_{y}\right) d s .
\end{aligned}
$$

(2.10) The 3rd term of R.H.S. of (2.5)

$$
\begin{aligned}
& =\int_{\gamma}\left\{\left(f x^{\prime}\right)^{\prime} \frac{\partial}{\partial x} \log \varphi+\left(f y^{\prime}\right)^{\prime} \frac{\partial}{\partial y} \log \varphi\right\} \varphi d s \\
& =\int_{\gamma}\left\{\left(f x^{\prime}\right)^{\prime} \varphi_{x}+\left(f y^{\prime}\right)^{\prime} \varphi_{y}\right\} d s .
\end{aligned}
$$

(2.11) The 4th term of R.H.S. of (2.5)

$$
\begin{aligned}
& =\int_{\gamma}\left(y^{\prime} \frac{\partial}{\partial x}-x^{\prime} \frac{\partial}{\partial y}\right)\left(\left.\frac{\partial}{\partial x} \log \varphi \cdot \frac{d}{d t}\right|_{t=0} \bar{x}+\left.\frac{\partial}{\partial y} \log \varphi \cdot \frac{d}{d t}\right|_{t=0} \bar{y}\right) \varphi d s \\
& =\int_{\gamma}\left(y^{\prime} \frac{\partial}{\partial x}-x^{\prime} \frac{\partial}{\partial y}\right)\left(-\frac{\partial}{\partial x} \log \varphi \cdot f y^{\prime}+\frac{\partial}{\partial y} \log \varphi \cdot f x^{\prime}\right) \varphi d s
\end{aligned}
$$




$$
\begin{gathered}
=\int_{\gamma}\left\{-f \cdot\left(y^{\prime}\right)^{2} \frac{\partial^{2}}{\partial x^{2}} \log \varphi-f \cdot\left(x^{\prime}\right)^{2} \frac{\partial^{2}}{\partial y^{2}} \log \varphi\right. \\
\left.+2 \cdot f\left(x^{\prime} y^{\prime}\right) \frac{\partial^{2}}{\partial x \partial y} \log \varphi\right\} \varphi d s .
\end{gathered}
$$

Combining (2.5), (2.8), .., and (2.11), by an easy calculation, we obtain

$$
\begin{aligned}
\left.\frac{d}{d t}\right|_{t=0} \int_{M} n H(t) d v & \\
=\int_{\gamma} & {\left[-2 f\left\{\left(x^{\prime \prime}\right)^{2}+\left(y^{\prime \prime}\right)^{2}\right\} \varphi+3 f\left(x^{\prime} y^{\prime \prime}-y^{\prime} x^{\prime \prime}\right)^{2} \varphi+f\left\{-\left(y^{\prime}\right)^{2} \frac{\partial^{2}}{\partial x^{2}} \log \varphi\right.\right.} \\
& \left.\left.-\left(x^{\prime}\right)^{2} \frac{\partial^{2}}{\partial y^{2}} \log \varphi+2\left(x^{\prime} y^{\prime}\right) \frac{\partial^{2}}{\partial x \partial y} \log \varphi\right\} \varphi-f \cdot(n H)^{2} \cdot \varphi\right] d s \\
=\int_{\gamma} & {\left[f\left(x^{\prime} y^{\prime \prime}-y^{\prime} x^{\prime \prime}\right)^{2} \varphi+f\left\{-\left(y^{\prime}\right)^{2} \frac{\partial^{2}}{\partial x^{2}} \log \varphi-\left(x^{\prime}\right)^{2} \frac{\partial^{2}}{\partial y^{2}} \log \varphi\right.\right.} \\
& \left.\left.+2\left(x^{\prime} y^{\prime}\right) \frac{\partial^{2}}{\partial x \partial y} \log \varphi\right\} \varphi-f \cdot(n H)^{2} \cdot \varphi\right] d s
\end{aligned}
$$

Comparing this equation with (2.2), we get

$$
\begin{aligned}
S= & (n H)^{2}-\left(x^{\prime} y^{\prime \prime}-y^{\prime} x^{\prime \prime}\right)^{2} \\
& +\left(y^{\prime}\right)^{2} \frac{\partial^{2}}{\partial x^{2}} \log \varphi-2\left(x^{\prime} y^{\prime}\right) \frac{\partial^{2}}{\partial x \partial y} \log \varphi+\left(x^{\prime}\right)^{2} \frac{\partial^{2}}{\partial y^{2}} \log \varphi .
\end{aligned}
$$

Let $\lambda_{1}=x^{\prime} y^{\prime \prime}-y^{\prime} x^{\prime \prime}, \lambda_{2}, \ldots, \lambda_{n}$ be the principal curvatures of $M_{\gamma}$. From (2.1) we get

$$
\lambda_{2}+\cdots+\lambda_{n}=-\frac{\partial}{\partial v_{\xi}} \log \varphi .
$$

Plug this into (2.13) we obtain

$$
\begin{aligned}
S= & \left(x^{\prime} y^{\prime \prime}-y^{\prime} x^{\prime \prime}-\frac{\partial}{\partial v_{\xi}} \log \varphi\right)^{2}-\left(x^{\prime} y^{\prime \prime}-y^{\prime} x^{\prime \prime}\right)^{2} \\
& +\left(y^{\prime}\right)^{2} \frac{\partial^{2}}{\partial x^{2}} \log \varphi-2\left(x^{\prime} y^{\prime}\right) \frac{\partial^{2}}{\partial x \partial y} \log \varphi+\left(x^{\prime}\right)^{2} \frac{\partial^{2}}{\partial y^{2}} \log \varphi . \\
= & -2\left(x^{\prime} y^{\prime \prime}-y^{\prime} x^{\prime \prime}\right) \frac{\partial}{\partial v_{\xi}} \log \varphi+\left(\frac{\partial}{\partial v_{\xi}} \log \varphi\right)^{2}+\frac{\partial^{2}}{\partial v_{\xi}^{2}} \log \varphi,
\end{aligned}
$$

which proves Lemma 2.3. 


\section{Proof}

Suppose $M_{\gamma}$ is a generalized rotational closed immersed hypersurface of cohomogeneity 2 with constant scalar curvature $S$. Since $M_{\gamma}$ is compact, it is easy to see that $S>0$. Let $\lambda_{1}=x^{\prime} y^{\prime \prime}-y^{\prime} x^{\prime \prime}, \lambda_{2}, \ldots, \lambda_{n}$ be the principal curvatures of $M_{\gamma}$. From (2.1) we get

$$
\lambda_{2}+\cdots+\lambda_{n}=-\frac{\partial}{\partial v_{\xi}} \log \varphi .
$$

From Lemma 2.3 , by using $S=2 \sum_{i<j} \lambda_{i} \lambda_{j}$ we obtain

$$
\lambda_{2}^{2}+\cdots+\lambda_{n}^{2}=-\frac{\partial^{2}}{\partial v_{\xi}^{2}} \log \varphi
$$

If there is a point such that $\frac{\partial}{\partial v_{\xi}} \log \varphi=0$, by (2.3) we obtain $S=\frac{\partial^{2}}{\partial v_{\xi}^{2}} \log \varphi$ at the point. From (3.2) we get $S \leq 0$, which contradicts with the assumption $S>0$. Thus we have proved the following lemma.

LEMMA 3.1. If $M_{\gamma}$ is a compact immersed hypersurface with constant scalar curvature $S$, then $\frac{\partial}{\partial v_{\xi}} \log \varphi$ never vanishes.

We examine the condition $\frac{\partial}{\partial v_{\xi}} \log \varphi \neq 0$ for 14 types of cohomogeneity 2 orthogonal transformation groups $\left(G, \mathbf{R}^{n+1}\right)$ [4], separately.

1) $\left(G, \mathbf{R}^{n+1}\right)=\left(S O(n-1), \mathbf{R}^{n+l}\right)$.

It is easy to see that a compact $S O(n-1)$-invariant hypersurface with constant scalar curvature is a round sphere (see, for example, [6]).

2) $\left(G, \mathbf{R}^{n+1}\right)=\left(S O(m) \times S O(l), \mathbf{R}^{m+l}\right)$.

Since $\varphi=c x^{m-1} y^{l-1}$, we have

$$
\begin{aligned}
\frac{\partial}{\partial v_{\xi}} \log \varphi & =-(m-1) \frac{y^{\prime}}{x}+(l-1) \frac{x^{\prime}}{y} \\
& =-(m-1) \frac{\sin \alpha}{x}+(l-1) \frac{\cos \alpha}{y},
\end{aligned}
$$

where we set $\left(x^{\prime}, y^{\prime}\right)=(\cos \alpha, \sin \alpha)$. Now we put $y / x=\tan \theta$. Then $0<\theta$ $<\pi / 2$. From (3.3) the condition $\frac{\partial}{\partial v_{\xi}} \log \varphi=0$ becomes

$$
C:(l-1) \cos \alpha \cos \theta-(m-1) \sin \alpha \sin \theta=0 .
$$

It is easy to see that each connected component of $C$ is a graph over $\theta$-axis and each connected component of $C$ is $\pi$ apart in the vertical direction (see Figure 1). If $\gamma$ has a self-intersection $\gamma(a)=\gamma(b)$, then the total change of $\alpha$ from $\gamma(a)$ 

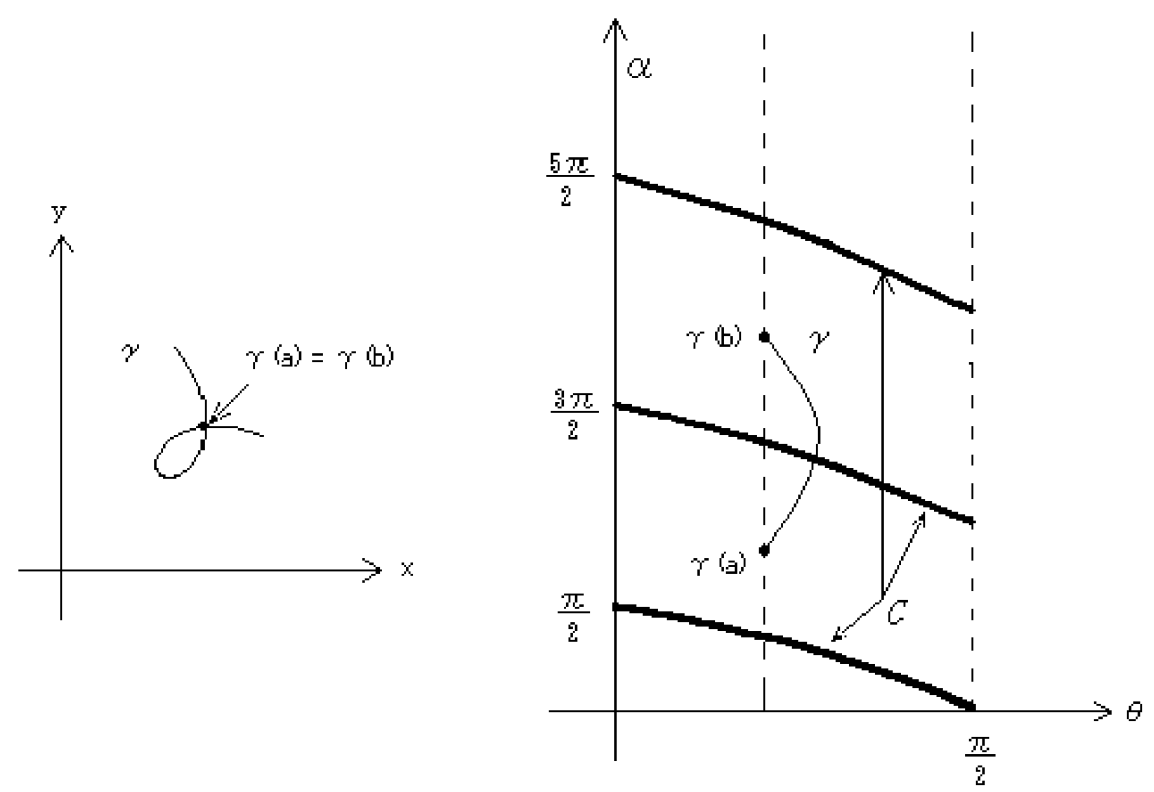

FIGURE 1

to $\gamma(b)$ is greater than or equal to $\pi$. Therefore $\gamma$ must intersect with some connected component of $C$ (see Figure 1). This contradicts with Lemma 3.1. 3) $(G, \Phi)=\left(S O(3), S^{2} \rho_{3}-1\right),(S U(3), A d),\left(S p(3), \Lambda^{2} v_{3}-1\right)$, or $\left(F_{4}, 1\right)$.

Then $\varphi=c\left\{y\left(3 x^{2} / 4-y^{2} / 4\right)\right\}^{k}$ for $k=1,2,4,8$, respectively.

$$
\frac{1}{k} \frac{\partial}{\partial v_{\xi}} \log \varphi=-\sin \alpha \cdot \frac{6 x}{3 x^{2}-y^{2}}+\cos \alpha \cdot \frac{1}{y}-\cos \alpha \cdot \frac{2 y}{3 x^{2}-y^{2}} .
$$

The condition $\frac{\partial}{\partial v_{\xi}} \log \varphi=0$ becomes

$$
C: \cos (\alpha+2 \theta)=0 \text {. }
$$

So the rest of the proof is the same as the case 2).

4) $(G, \Phi)=(S O(5), A d), \quad\left(S O(2) \times S O(m), \rho_{2} \otimes \rho_{m}\right), \quad(S(U(2) \times U(m))$, $\left.\left[\mu_{2} \otimes_{\mathbf{C}} \mu_{m}\right]_{\mathbf{R}}\right),\left(S p(2) \times S p(m), v_{-} 2 \otimes_{\mathbf{H}} v_{m}^{*}\right),\left(U(5),\left[\Lambda^{2} \mu_{5}\right]_{\mathbf{R}}\right)$ or $(U(1) \times \operatorname{Spin}(10)$, $\left.\left[\mu_{1} \otimes_{\mathbf{C}} \Delta_{1}^{+}\right]_{\mathbf{R}}\right)$.

In this case $\varphi=c(x y)^{m}\left(x^{2}-y^{2}\right)^{k}$ for some $m \in \mathbf{N}$ and some $k \geq 1$. The condition $\frac{\partial}{\partial v_{\xi}} \log \varphi=0$ becomes

$$
\begin{aligned}
& C: \sin \theta\left\{(m+2 k) \cos ^{2} \theta-m \sin ^{2} \theta\right\} \sin \alpha \\
& \quad-\cos \theta\left\{m \cos ^{2} \theta-(m+2 k) \sin ^{2} \theta\right\} \cos \alpha=0 .
\end{aligned}
$$

Set 


$$
\begin{aligned}
f(\theta, \alpha)= & \sin \theta\left\{(m+2 k) \cos ^{2} \theta-m \sin ^{2} \theta\right\} \sin \alpha \\
& -\cos \theta\left\{m \cos ^{2} \theta-(m+2 k) \sin ^{2} \theta\right\} \cos \alpha .
\end{aligned}
$$

Then

$$
\begin{aligned}
f_{\alpha}= & \sin \theta\left\{(m+2 k) \cos ^{2} \theta-m \sin ^{2} \theta\right\} \cos \alpha \\
& +\cos \theta\left\{m \cos ^{2} \theta-(m+2 k) \sin ^{2} \theta\right\} \sin \alpha .
\end{aligned}
$$

Suppose that $f=f_{\alpha}=0$. Since $(\sin \alpha,-\cos \alpha),(\cos \alpha, \sin \alpha)$ are linearly independent, we obtain

$$
\sin \theta\left((m+2 k) \cos ^{2} \theta-m \sin ^{2} \theta\right)=\cos \theta\left(m \cos ^{2} \theta-(m+2 k) \sin ^{2} \theta\right)=0 .
$$

Since $0<\theta<\pi / 2$, we obtain $(m+2 k) \cos ^{2} \theta-m \sin ^{2} \theta=0, \quad m \cos ^{2} \theta-$ $(m+2 k) \sin ^{2} \theta=0$. Thus $m+2 k=m$. This contradicts with the assumption $k \geq 1$. Therefore each connected component of $C$ is a graph over $\theta$-axis and is $\pi$ apart in the vertical direction. The rest of the proof is the same as 2).

5) $(G, \Phi)=\left(G_{2}, A d\right)$ or $\left(S O(4), \stackrel{1}{\circ} \square_{0}^{3}\right)$.

In this case $\varphi=c\left\{x y\left(3 x^{2} / 4-y^{2} / 4\right)\left(x^{2} / 4-3 y^{2} / 4\right)\right\}^{k}$ for $k=2,1$. The condition $\frac{\partial}{\partial v_{\xi}} \log \varphi=0$ becomes

$$
\begin{aligned}
C: & \left\{5 \cos ^{4} \theta-10 \cos ^{2} \theta \sin ^{2} \theta+\sin ^{4} \theta\right\} \sin \theta \sin \alpha \\
& \quad-\left\{\cos ^{4} \theta-10 \cos ^{2} \theta \sin ^{2} \theta+5 \sin ^{4} \theta\right\} \cos \theta \cos \alpha=0 .
\end{aligned}
$$

It is easy to see that each connected component of $C$ is also a graph over $\theta$-axis, and this finishes the proof of Theorem 1.1.

\section{Remarks.}

1. By using Lemma 2.3, we can compute constant scalar curvature equations for generalized rotational hypersurfaces of cohomogeneity 2. By analyzing these ODE systems we can construct noncompact complete hypersurfaces with constant scalar curvature in the Euclidean spaces. We will discuss this in the future. See [7] for the most easy case.

2. For construction of complete hypersurfaces with 0 scalar curvature, see the works by Palmas [8] and Sato [12].

\section{REFERENCES}

[1] A. D. Aleksandrov, Uniqueness theorems for surfaces in the large, Vestnik Leningrad Univ. Math. 13 (1958), 5-8.

[2] Q.-M. Cheng, Complete hypersurfaces in a Euclidean space $\mathbf{R}^{n+1}$ with constant scalar curvature, Indiana Univ. Math. J. 51 (2002), 53-68.

[3] W. Y. Hsiang, Generalized rotational hypersurfaces of constant mean curvature in the Euclidean spaces. I, J. Differential Geom. 17 (1982), 337-356. 
[4] W. Y. Hsiang and H. B. Lawson, Minimal submanifolds of low cohomogeneity, J. Differential Geometry 5 (1971), 1-38.

[5] W. Y. Hsiang, Z. H. Teng and W. C. Yu, New examples of constant mean curvature immersions of $(2 k-1)$-spheres into Euclidean $2 k$-space, Ann. of Math. 117 (1983), 609625.

[6] M. L. LeITE, Rotational hypersurfaces of space forms with constant scalar curvature, Manuscripta Math. 67 (1990), 285-304.

[7] T. OKayasu, $O(2) \times O(2)$-invariant hypersurfaces with constant negative scalar curvature in $E^{4}$, Proc. Amer. Math. Soc. 107 (1989), 1045-1050.

[8] O. Palmas, $O(2) \times O(2)$-invariant hypersurfaces with zero scalar curvature, Arch. Math. (Basel) 74 (2000), 226-233.

[9] R. C. ReIlly, Variational properties of functions of mean curvatures for hypersurfaces in space forms, J. Differential Geom. 8 (1973), 465-477.

[10] A. Ros, Compact hypersurfaces with constant scalar curvature and a congruence theorem, with an appendix by Nicholas J. Korevaar, J. Differential Geom. 27 (1988), 215-223.

[11] H. Rosenberg, Hypersurfaces of constant curvature in space forms, Bull. Sci. Math. 117 (1993), 211-239.

[12] J. SAто, $O(p+1) \times O(p+1)$-invariant hypersurfaces with zero scalar curvature in Euclidean space, An. Acad. Brasil. Cienc. 72 (2000), 109-115.

[13] H. C. Wente, Counterexample to a conjecture of H. Hopf, Pacific J. Math. 121 (1986), 193243.

[14] S.-T. YAU, Seminar on Differential Geometry, Problem Section, Ann. of Math. Studies 102, Princeton, 1982, 669-706.

\author{
Department of Mathematics \\ FACULTy OF EDUCATION \\ IBARAKI UNIVERSITY \\ Mito, IBARAKI 310-8512 \\ JAPAN \\ E-mail: okayasu@mx.ibaraki.ac.jp
}

